\title{
Generation Revenue Regulation and Incentive Contract Design in Electricity Market
}

\author{
Baike Chen ${ }^{1, *}$, Yuxia Rong ${ }^{1}$, and Zhaoxia Jing ${ }^{1}$ \\ ${ }^{1}$ South China University of Technology, School of Electric Power, Guangzhou, China
}

\begin{abstract}
In order to curb market power, encourage investment and redistribute welfare, revenue regulation should be carried out in electricity market. The incentive contract represented by the Contract for Difference is a kind of regulation. This paper proposes a Government Authorized Contract based on Revenue Estimation Method (REM) from the perspective of regulating generation revenue. First, the principle and design of the Vesting Contract in Singapore electricity market is employed, analyzing its limitations in regulation. Then, the incentive contract based on REM is presented in four steps. Finally, the IEEE30 node classic system is adopted to demonstrate the proposed model, showing that it can promote generators to bid rationally.
\end{abstract}

\section{Introduction}

Generation revenue regulation is the core content of Electricity market regulation. In theory, revenue regulation should be relaxed after the introduction of competition, so that the wholesale market price can truly reflect the social cost and achieve the goal of optimal allocation of social resources. However, the electricity market is an oligopoly market, which is constrained by network economy, real-time balance, scale economy and information asymmetry. The simple market mechanism is easy to induce the exercise of market power, and sometimes can not guarantee the safety and reliability of the system, so we must rely on the revenue regulation. The construction of electricity market is actually an evolution from high regulation to light regulation, during which the government and power generation enterprises will face different information structures, constraints and feasible tools, and need to solve the problems of moral hazard and adverse selection.

The government should take incentive measures to regulate the revenue of the generation in order to promote the healthy development of the market. According to whether transfer payment is allowed or not, incentive schemes can be divided into price regulation and government procurement contract regulation. The former has no transfer payment and is mainly applied to most regulated private enterprises, which can be divided into two modes: rate-of-return regulation (ROR), mainly used in the U.S. electricity market, and perfermance based regulation (PBR), mainly used in European electricity market. The latter directly subsidizes public enterprises by signing contracts with the government, so as to restrain market power, subsidize high-cost units, and dredge the cost of power generation.
At present, scholars at home and abroad have carried out extensive research on revenue regulation in electricity market. In terms of ROR, literature [1] proposed that ROR lacks incentives to promote cost reduction and tends to encourage excessive capital investment. In terms of PBR, literature [2] examined the magnitude of the capital bias and its welfare effects by means of numerical simulations. The result shows that the Nordic revenue cap decreases the monopoly profit and hence increases consumer surplus compared to the unregulated monopoly. Literature [3] presented a basic model of a regulated firm that operates under a vertically separated market structure to show that when the demand elasticity is large, the price rise degree under the Revenue-cap regulation is greater than that under the Price-cap regulation. In addition, reference [4] proposed a Profit-cap regulation method, which constructs profit space by dividing the comprehensive cost into sunk cost and activity cost, so as to promote cost reduction and efficiency. In terms of government procurement contract regulation, most countries, such as Singapore, Ontario of Canada and Zhejiang of China, sign Government Authorized Contract for Difference (CfD) with power generation in the early stage of market construction to recover stranded costs[5], restrain market power[6] and guarantee capacity adequacy. However, there are few literatures on detailed design of incentive contracts.

In fact, most of the literatures focus on price regulation rather than incentive contract design. This paper is divided into four parts. The first part is introduction and literature review. The second part briefly introduces the incentive $\mathrm{CfD}$ in the electricity market of Singapore and Ontario, Canada. The third part verifies the advantages of the $\mathrm{CfD}$ through examples. The fourth part is our conclusion.

\footnotetext{
* Corresponding author: epchenbaike@mail.scut.edu.cn
} 


\section{Incentive Contract}

\subsection{Vesting Contract in National Electricity Market of Singapore}

In the early stage of market construction, the top three power producers in Singapore controlled $85 \%$ of Singapore's total installed capacity. They controlled the electricity price at the maximum price set by the Energy Market Authority (EMA) through capacity withholding, which led to a surge in electricity price during the peak period. In order to curb the market power and maintain the stability of electricity price, EMA introduced the vesting contract in 2004, which is one of the CfD, compulsorily signed by Market Support Services Licensee with generators. The contract volume undertaken by the generators is settled according to the contract price and is not affected by the spot price. The revenue of contract affected by the contract quantity, contract price and benchmark price[7].

$$
\begin{aligned}
R_{i} & =R_{i}^{\mathrm{C}}+R_{i}^{\mathrm{spot}} \\
& =\sum_{t \in T}\left(P_{i}^{\mathrm{C}}-P_{i, t}^{\mathrm{spot}}\right) \cdot Q_{i, t}^{\mathrm{C}}+\sum_{t \in T} P_{i, t}^{\mathrm{spot}} \cdot Q_{i, t}^{\mathrm{spot}} \\
& =\sum_{t \in T}\left[P_{i}^{\mathrm{C}} \cdot Q_{i, t}^{\mathrm{C}}+P_{i, t}^{\mathrm{spot}} \cdot\left(Q_{i, t}^{\mathrm{spot}}-Q_{i, t}^{\mathrm{C}}\right)\right]
\end{aligned}
$$

where $R_{i}, R_{i}^{\mathrm{C}}$ and $R_{i}^{\text {spot }}$ denote total revenue, contract revenue and spot market revenue of unit $i$ respectively. $P_{i}^{\mathrm{C}}$ and $Q_{i, t}^{\mathrm{C}}$ denote the price and the amount of the CfD for the the unit $i$ in interval $t$ th. $P_{i, t}^{\text {spot }}$ denotes the clearing price in the spot market in interval $t$ th, that is, the benchmark price. $Q_{i, t}^{\text {spot }}$ represents the clearing capacity of the unit $i$ in interval $t$ th.

It can be seen from formula (1) that when the $P_{i}^{\mathrm{C}}$ is determined, the main factor affecting the units' revenue is $Q_{i, t}^{\mathrm{C}}$, which needs to be decomposed to all settlement interval by decomposition algorithm after the total contract volume is determined. It is not conducive to regulate the revenue of generators to allocate too little contract amount during peak-load period or too much contract amount during low-load period[8].

\subsection{Clean Energy Supply Contract in Ontario electricity market}

The Ontario electricity market regulates generators' revenue and guarantees capacity adequacy through Clean Energy Supply Contract (CES) based on Revenue Estimation Method (REM). Different from the traditional $\mathrm{CfD}$, this contract does not need to be decomposed[9]. Meanwhile, the bidding system of power generation rights is introduced to strengthen the contract competition and promote the cost reduction and efficiency.

\subsubsection{Basic principle}

The settlement of CES contract is based on the Imputed Gross Energy Market Revenues (IGEMR) and the Imputed Variable Energy Cost (IVEC) so as to calculate the Imputed Net Revenue (INR), which is used to offset the Total Monthly Fixed Capacity Payment (TMFCP). The deficiency is made up by the Contingent Support Payment (CSP), and the excess, as the Revenue Sharing Payment (RSP), shall be turned over to the Independent Electricity System Operator(IESO), as shown in Figure 1.

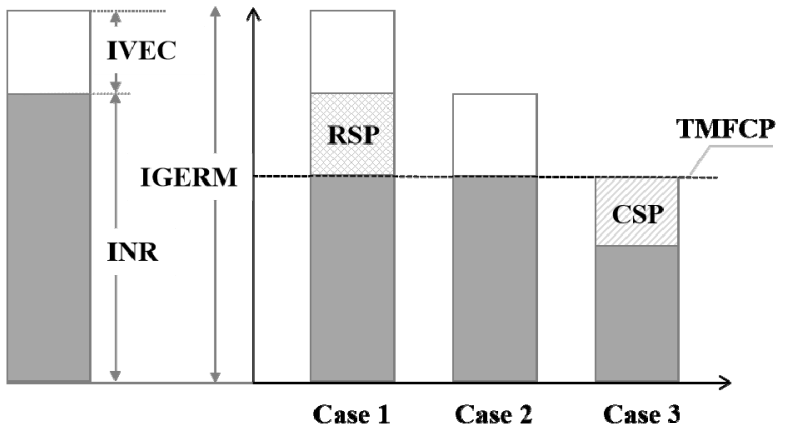

Fig. 1. Settlement of the CES.

INR and TMFCP are the final factors that affect the contract revenue. The REM calculates the contract payment through five steps, as shown in Figure 2.

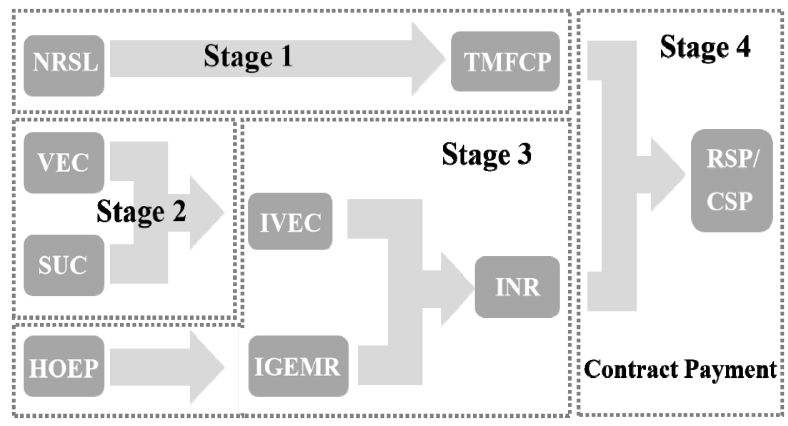

Fig. 2. Settlement of the CES.

\subsubsection{Stage 1}

TMFCP $R^{\mathrm{TMF}}$ is calculated from the Contract Capacity $C^{\mathrm{C}}$, which is adjusted according to the effectiveness of the capacity, and the Net Revenue Support Level (NRSL) $R_{y}^{\mathrm{NRL}}$.

$$
R^{\mathrm{TMF}}=C^{\mathrm{ACC}} R_{y}^{\mathrm{NRL}}=\alpha C^{\mathrm{C}} R_{y}^{\mathrm{NRL}}
$$

where $C^{\mathrm{ACC}}$ is the Adjusted Contract Capacity (in MW) for the Settlement Month. $\alpha$ denotes the Capacity Reduction Factor for the Settlement Month, that is determined by the IESO through the Capacity Check Test every year. If the unit passes the test, $\alpha$ is 1.0. If it is in $80 \%-100 \%$, the $\alpha$ will be taken as the corresponding score. Otherwise, the unit will be deemed as breach of contract. In addition, $R_{y}^{\mathrm{NRL}}$ adopt the agreed value $R_{\mathrm{b}}^{\mathrm{NRL}}$ in the first year, and then are adjusted 
according to the CPI of the previous year in subsequent years.

$$
R_{y}^{\mathrm{NRL}}=R_{\mathrm{b}}^{\mathrm{NRL}} \times \beta \times \frac{I_{y}^{\mathrm{CPI}}}{I_{\mathrm{b}}^{\mathrm{CPI}}}+R_{\mathrm{b}}^{\mathrm{NRL}} \times(1-\beta)
$$

where $I_{\mathrm{b}}^{\mathrm{CPI}}$ and $I_{y}^{\mathrm{CPI}}$ are the CPI on the contract signing date and the contract effective date respectively. $\beta$ is the Net Revenue Requirement Indexing Factor expressed as a decimal figure between 0.00 and 0.20 .

\subsubsection{Stage 2}

IVEC is established daily and is used in a couple of places: a) Determining Imputed Production Intervals in the day, and b) Determining Imputed Variable Energy Cost in the month, which includes Variable Energy Cost (VEC) $C^{\mathrm{VEC}}$ and Start-up Cost (SUC) $C_{d}^{\mathrm{SUC}}$.

The calculation formula of $C^{\mathrm{VEC}}$ and $C_{d}^{\mathrm{SUC}}$ are as follows:

$$
\begin{gathered}
C_{d}^{\mathrm{VEC}}=P_{d}^{\mathrm{GAS}} \times \delta^{\mathrm{HEAT}}+C_{y}^{\mathrm{O} \& \mathrm{M}} \\
C_{y}^{\mathrm{O} \& \mathrm{M}}=C_{\mathrm{b}}^{\mathrm{O} \& \mathrm{M}} \times \frac{I_{y}^{\mathrm{CPI}}}{I_{\mathrm{b}}^{\mathrm{CPI}}} \\
C_{d}^{\mathrm{SUC}}=P_{d}^{\mathrm{GAS}} \times Q_{d}^{\mathrm{SUC}}
\end{gathered}
$$

where $P_{d}^{\mathrm{GAS}}$ denotes the natural gas price, which is the "day ahead" price on Union Dawn Daily Spot Gas Price Index; $\delta^{\text {HEAT }}$ is the Specified Heat Rate agreed on the contract signing date. $C_{y}^{\mathrm{O \& M}}$ includes an amount that reflects the variable portion of gas transmission/distribution cost, which adopt the agreed value in the first year, and then are adjusted according to the CPI of the previous year in subsequent years. $C_{d}^{\text {SUC }}$ represents the cost of gas required for one start-up. $Q_{d}^{\text {SUC }}$ represents the volume of natural gas for one startup, agreed on the contract signing date.

\subsubsection{Stage 3}

The calculation formula of INR $R_{m}^{\mathrm{INR}}$ is as follows:

$$
\begin{gathered}
R_{m}^{\mathrm{INR}}=R_{m}^{\mathrm{IGEMR}}-C_{m}^{\mathrm{IVEC}} \\
R_{m}^{\mathrm{IGEMR}}=\sum_{h=1}^{H_{\mathrm{m}}} C^{\mathrm{ACC}} \cdot P_{h} \\
C_{m}^{\mathrm{IVEC}}=\sum_{d=1}^{m}\left[C_{d}^{\mathrm{SUC}} \cdot N_{d}^{\mathrm{SUC}}+\sum_{h=1}^{H_{\mathrm{d}}}\left(C_{d}^{\mathrm{VEC}} \cdot C^{\mathrm{ACC}}\right)\right]
\end{gathered}
$$

where $R_{m}^{\mathrm{IGEMR}}$ and $C_{m}^{\mathrm{IVEC}}$ denote the the Imputed Gross Energy Market Revenues and the Imputed Variable Energy Cost respectively. $P_{h}$ denotes the spot market clearing price in interval $h$ th. $H$ denotes the estimated total number of hours of production for the generator. When the spot market clearing price is higher than the Imputed Variable Energy Cost, it is considered that the unit should be in the state of power generation. $N_{d}^{\mathrm{SUC}}$ represents the number of starts of the unit in a day.

\subsubsection{Stage 4}

The monthly contract settlement method is as follows:

$$
R_{m}^{\mathrm{c}}=\left\{\begin{array}{cl}
R_{m}^{\mathrm{CSP}} & R^{\mathrm{TMF}} \geq R_{m}^{\mathrm{INR}} \\
-R_{m}^{\mathrm{RSP}} & R^{\mathrm{TMF}}<R_{m}^{\mathrm{INR}}
\end{array}\right.
$$

where $R_{m}^{\mathrm{c}}$ is the contract revenue of the generator. $R_{m}^{\mathrm{CSP}}$ and the $R_{m}^{\mathrm{RSP}}$ denote the the Contingent Support Payment and the Revenue Sharing Payment respectively.

$$
\begin{gathered}
R_{m}^{\mathrm{CSP}}=R^{\mathrm{TMF}}-R_{m}^{\mathrm{INR}} \\
R_{m}^{\mathrm{RSP}}=0.95\left(R_{m}^{\mathrm{INR}}-R^{\mathrm{TMF}}\right)
\end{gathered}
$$

\section{Case Studies}

\subsection{Date of the Case Studies}

In this section, the IEEE30 node classic system with 6 generators, 20 loads, 41 transmission lines is employed to demonstrate the model of the REM. The total installed capacity of six generating units is $2150 \mathrm{MW}$, among that three gas-fired units with an installed capacity of $600 \mathrm{MW}$, accounting for $27.9 \%$, and the remaining three coal-fired units with an installed capacity of $1550 \mathrm{MW}$, accounting for $72.1 \%$, as shown in Table I. Unit 4 is the subsidized unit with Government Authorized Contract. The contract capacity is $200 \mathrm{MW}$, the Net Revenue Support Level is $400 \mathrm{RMB} / \mathrm{MW} /$ day, the Variable Energy Cost is $600 \mathrm{RMB} / \mathrm{MWh}$, and the Start-up Cost is $3000 \mathrm{RMB} /$ time. The demand function is formulated based on a typical load curve with a peak load of 2025MW. Specially, the time scale of the spot market price is hour. We changed the unit 4 price from $550 \mathrm{RMB} / \mathrm{MWh}$ to $700 \mathrm{RMB} / \mathrm{MWh}$.

Table 1. Basic parameters of generators.

\begin{tabular}{|c|c|c|c|}
\hline Type & Unit & Capacity(MW) & $\operatorname{Cost(RMB/MW)~}$ \\
\hline \multirow{3}{*}{$\begin{array}{c}\text { coal- } \\
\text { fired }\end{array}$} & 1 & 450 & 270 \\
\cline { 2 - 4 } & 3 & 500 & 250 \\
\cline { 2 - 4 } & 5 & 600 & 220 \\
\hline \multirow{3}{*}{$\begin{array}{c}\text { gas- } \\
\text { fired }\end{array}$} & 2 & 200 & 650 \\
\cline { 2 - 4 } & 4 & 200 & 600 \\
\hline
\end{tabular}

\subsection{Results and Discussions}

\subsubsection{Changes in contract revenue}

After running the model, the subsidy revenue and relevant details of the unit 4 can be obtained. Firstly, the Total Monthly Fixed Capacity Payment of the unit is not related to the market clearing price, but only related to the Net Revenue Support Level and Contract Capacity, so the TMFC remains unchanged. Secondly, the Imputed 
Gross Energy Market Revenues is related to market clearing price and Contract Capacity. When the market price is higher than the marginal cost of unit 4 approved by the government, the unit 4 is considered to be generating according to the contract capacity. At this time, the Imputed Variable Energy Cost of the unit needs to be calculated. Hence, the decrease of unit quotation does not affect the Imputed Net Revenue, but the increase of unit quotation will increase both Imputed Gross Energy Market Revenues and Imputed Variable Energy Cost, and the IGEMR changes more greatly than the IEVC, resulting in the increase of INR. As a result, if the unit is not quoted at cost, the contract revenue will be reduced, as shown in Figure 3.

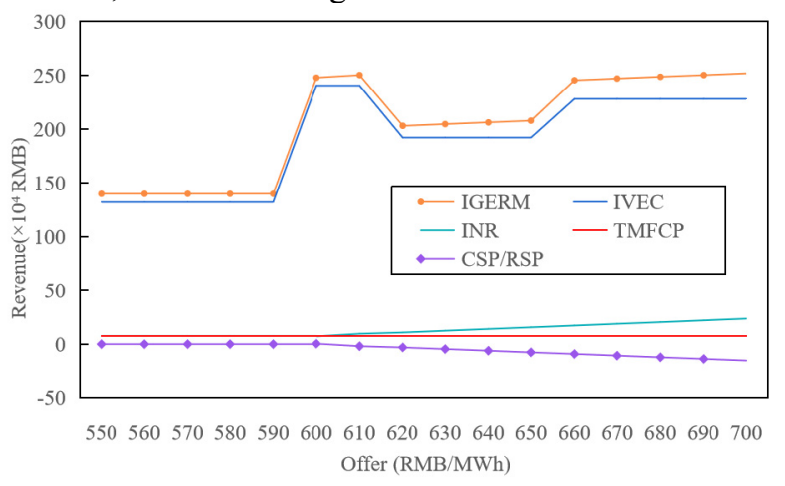

Fig. 3. The change of contract revenue with the change of quotation.

\subsubsection{Changes in total revenue}

The main parameters affecting the revenue of unit 4 are spot market revenue and contract revenue, as shown in Figure 4.

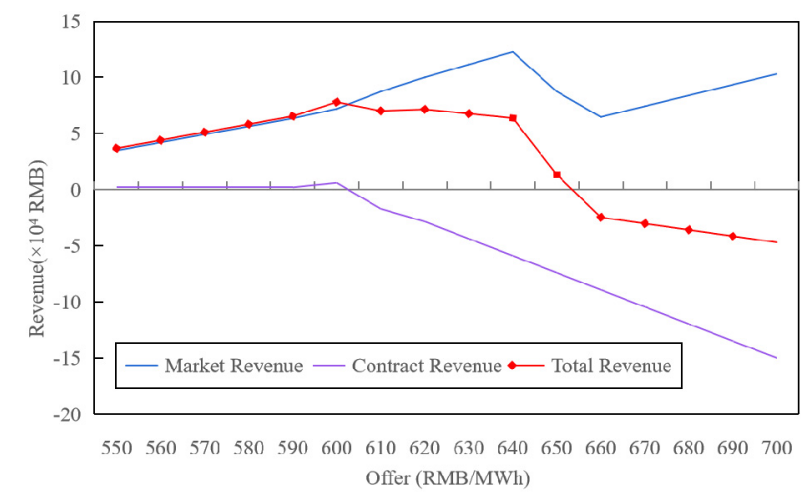

Fig. 4. The change of total revenue with the change of quotation.

a) When the quoted price of unit 4 is lower than its' cost, although the market clearing volume increases, the power generation is at a loss, and the market revenue decreases. According to ection 3.2.1, when the quoted price is lower than the marginal cost, the contract revenue of unit 4 changes little, or even no change, so the total revenue decreases;

b) When the quoted price of unit 4 is higher than its' cost, the market clearing price rises, making the market revenue rise. According to ection 3.2.1, when the unit 4 price is higher than the marginal cost, the contract revenue decreases, or even down to a negative value, so the total revenue decreases.

It can be observed that the total revenue of unit 4 is maximized when quoted at marginal cost. The proposed model can encourage the unit to quote base on its' marginal cost, playing a very good role in revenue regulation.

\section{Conclusion}

Revenue regulation of electricity market can supervise generators' performance, and the government procurement contract is one of the way of revenue regulation, which can realize the redistribution of social welfare. Firstly, the regulation methods in power market is introduced. Secondly the CfD is presented based on the Singapore power market to illustrate its limitations. Then a model of incentive contract based on the REM is proposed, from the perspective of regulating the revenue and mitigating market power. Finally, the performance of the developed model is assessed through the IEEE30 node classic system. Numerical results demonstrate that the proposed model can well regulate the revenue of power generators, leading to athe reasonable quotation of market members and realizing the rational redistribution of social welfare.

\section{References}

1. H. Averch, L.L. Johnson. Behavior of the fifirm under regulatory constraint. Am. Econ. Rev, 52 (5), 1052-1069(1962).

2. A. Campbell. Cap prices or cap revenues? The dilemma of electric utility networks. Energy Economics, 74, 802-812(2018).

3. T. Kuosmanen, T. Nguyen. Cap prices or cap revenues? The dilemma of electric utility networks. Energy Policy, 139(2020).

4. W. Xuehao, Z. Hao. Implementation of Transform From Price Capping to Profit Capping. Power System Technology, 27(5)(2003).

5. K. Edward D. Vesting contracts: a tool for electricity market transition. The Electricity Journal, 14(6), 11-22(2001).

6. E. Hausman, R. Hornby, A. Smith. Bilateral contracting in deregulated electricity markets. American Public Power Association(2008).

7. L. Ziya, J. Tianyao, J. Zhaoxia, H. Aimin. Design and Application of Contract for Difference in Electricity Market. Power System Technology, 43(8)(2019).

8. X. Chengwei, P. Kaiyuan, W. Fushuan, G. Jianrong, et al. Decomposition Model of Contract for Difference Considering Market Power Mitigation. $\operatorname{IEEE}(2020)$.

9. Z. Jisong, C. Baike, J. Tianyao, J. Zhaoxia. Transitional Revenue Scheme for High-cost 
Generator Unit in Guangdong Electricity Market Based on Government Authorized Contracts. CSEE (2020). 